\title{
Adipositas: State of the Art
}

\author{
Martina de Zwaan Astrid Müller \\ Klinik für Psychosomatik und Psychotherapie, Medizinische Hochschule Hannover, Deutschland
}

\section{Schlüsselwörter}

Adipositas · Epidemiologie · Ätiologie · Therapie .

Komorbidität $\cdot$ Stigmatisierung $\cdot$ Gewichtsstabilisierung

\section{Zusammenfassung}

Adipositas ist gemäß den WHO-Kriterien über einen BMI von $\geq 30 \mathrm{~kg} / \mathrm{m}^{2}$ definiert. Die Adipositasprävalenz hat in den letzten Jahren in Deutschland weiter zugenommen und liegt bei etwa $24 \%$ der Bevölkerung. Als ursächlich für die Zunahme der Adipositas werden bei zugrundeliegender genetischer Vulnerabilität vor allem die leichte Verfügbarkeit hochkalorischer Nahrungsmittel und die Bewegungsarmut in der Überflussgesellschaft angesehen. Die Grundlage jedes konservativen Gewichtsmanagements sollte ein Basisprogramm sein, das die Komponenten Ernährungs-, Bewegungs- und Verhaltenstherapie umfasst. Insgesamt sollten die Therapieziele realistisch sein und an individuelle Bedingungen angepasst werden. Dabei sollten individuelle Komorbiditäten, Risiken, Erwartungen und Ressourcen des Betroffenen stärker berücksichtigt werden und nicht nur die alleinige Gewichtsreduktion im Fokus stehen. Langfristiger Gewichtserhalt ist jedoch weiterhin schwer zu erreichen. Bei extremer Adipositas sind chirurgische Interventionen erfolgreich. Die psychische Komorbidität ist bei Menschen mit Adipositas erhöht, sowohl in der Bevölkerung als auch in klinischen Gruppen. Dies betrifft vor allem affektive Störungen, Angststörungen und Essstörungen (vor allem Binge-Eating-Störung). Auch ADHS ist mit Adipositas assoziiert, im Kindes- und Jugendlichen- wie im Erwachsenenalter. Adipositas wird in unserer Gesellschaft mehr stigmatisiert als andere körperliche und seelische Erkrankungen.

\section{Keywords}

Obesity · Epidemiology · Etiology · Treatment .

Comorbidity - Stigmatization - Weight loss maintenance

\section{Summary}

Obesity: State of the Art

According to WHO criteria obesity is defined as a BMI of $\geq 30 \mathrm{~kg} / \mathrm{m}^{2}$. In Germany the prevalence of obesity has increased over the last decades, affecting about $24 \%$ of the population. A genetic vulnerability combined with easy access to high calorie food and low levels of physical activity are seen as main reasons for this increase in prevalence rates in affluent societies. Weight loss programs should include the following components: nutrition, physical activity, and behavior modification. Overall, treatment goals should be realistic and should be adjusted to the individual's needs. Comorbidities, risks, expectancies, and resources of the individual should be considered; treatment should not only focus on weight loss. Long-term weight maintenance is still difficult to achieve. For severely obese individuals bariatric surgery is an effective treatment strategy. Individuals with obesity have an increased risk of mental comorbidity; this could be found in population-based as well as clinical samples. This includes affective disorders, anxiety disorders, and eating disorders (especially binge eating disorder). Also ADHD is associated with obesity in children, adolescents as well as in adults. In our society obesity is more stigmatized than other mental or somatic disorders.

\section{KARGER \\ Fax +497614520714 \\ Information@Karger.com}

www.karger.com (c) 2014 S. Karger GmbH, Freiburg

1016-6262/14/0242-0093\$39.50/0

Accessible online at:

www.karger.com/ver
Prof. Dr. med. Martina de Zwaan

Klinik für Psychosomatik und Psychotherapie

Medizinische Hochschule Hannover

Carl-Neuberg-Straße 1, 30625 Hannover, Deutschland

dezwaan.martina@mh-hannover.de 


\section{Epidemiologie}

Übergewicht und Adipositas stellen Herausforderungen für das Gesundheitssystem dar. Eine Adipositas liegt ab einem Body-Mass-Index (BMI) von $30 \mathrm{~kg} / \mathrm{m}^{2}$ vor und geht mit einem deutlich erhöhten Morbiditäts- und Mortalitätsrisiko einher, das mit zunehmendem BMI ansteigt. Eine rezente Repräsentativerhebung des Robert-Koch-Instituts hat gezeigt, dass 2010 67\% der Männer und 53\% der Frauen zwischen 18 und 79 Jahren übergewichtig und 23\% der Männer und $24 \%$ der Frauen adipös waren [Kurth, 2012]. In die Untersuchung wurden 7116 Personen eingeschlossen und Gewicht und Größe standardisiert erhoben. Im Vergleich zum Bundes-Gesundheitssurvey von 1998 hat sich der Anteil adipöser Männer in allen Altersgruppen, am meisten jedoch bei den jungen Männern, deutlich erhöht. 2 repräsentative Bevölkerungsumfragen 2009 und 2011 ergaben, dass etwa 1-2\% der erwachsenen deutschen Bevölkerung an Adipositas Grad III leiden (BMI $\geq 40 \mathrm{~kg} / \mathrm{m}^{2}$ ). In diesen Untersuchungen wurden die Teilnehmenden nach Gewicht und Größe gefragt, sodass die Prävalenz möglicherweise unterschätzt wurde [de Zwaan, 2012]. In den USA liegt die Prävalenz der Adipositas in keinem Bundesland unter 20\%. Insgesamt sind 35,7\% der Erwachsenen adipös. Mittlerweile wird nicht mehr nur von einer Adipositas-Epidemie, sondern von einer Adipositas-Pandemie gesprochen, da eine Zunahme der Adipositas weltweit zu verzeichnen ist.

Besonders alarmierend ist der mit 17\% sehr hohe Anzahl adipöser Kinder und Jugendlicher (2-19 Jahre) in den USA. In Deutschland sind laut Kinder- und Jugendsurvey 2006 [Rosario et al., 2010] 6,1\% der 3- bis 17-Jährigen adipös. Einschulungsdaten aus Erlangen und Umgebung ergaben eine Zunahme der Prävalenz von Übergewicht und Adipositas von 6,3 auf 9,5\% aller Kinder innerhalb von nur 5 Jahren von 1995/19962000/2001 [Knerr et al., 2005]. Es wurden insgesamt 4610 Kinder aus der Stadt Erlangen und dem Landkreis ErlangenHöchstadt untersucht. Der Anteil nichtdeutscher Kinder lag bei 6,1 bzw. 6,9\%, das mittlere Alter betrug 6,7 Jahre, 49\% waren weiblich. Experten zufolge soll sich die Kurve bei Kindern und Jugendlichen abflachen und sogar leicht rückläufig sein, wobei immer noch eine hohe Prävalenz besteht.

\section{Ätiologie}

Es existiert eine große Anzahl von Studien mit Zwillingen und Adoptivkindern, die den Einfluss genetischer Faktoren auf den BMI belegen [Wirth, 2008; Frieling et al., 2008]. So konnte gezeigt werden, dass der BMI von Adoptivkindern nicht mit dem ihrer Adoptiveltern korrespondiert, wohl aber mit dem BMI der leiblichen Eltern. Das Ausmaß des genetischen Einflusses wird von Experten mit einem Wert zwischen 50 und $60 \%$ angegeben [Wirth, 2008], wobei die Erblichkeitsschätzer bei Kindern und Jugendlichen höher sind als bei Er- wachsenen. Mutationen einzelner Gene, die zu Adipositas führen, sind selten und auf wenige Fälle beschränkt. Hierzu zählt auch die Mutation des Leptingens, bei der die Adipositas durch Leptinsubstitution ursächlich behandelt werden kann. Extrem adipöse Mausmutanten mit fehlendem Leptingen (ob-Maus) oder fehlendem Leptinrezeptor (db-Maus) wurden bereits in den 1940er Jahren in den Jackson Laboratories entdeckt. Schwere Adipositas ist zudem ein Symptom bei komplexeren genetischen Erkrankungen wie z.B. dem Prader-Willi-Syndrom. Insgesamt sind diese Mutationen selten und erklären die vorliegende Adipositas-Epidemie in den Industrienationen nicht. Die genetische Prädisposition für eine idiopathische Adipositas hat höchstwahrscheinlich in den meisten Fällen eine polygene Basis; hierbei hat jede einzelne Genvariante nur einen kleinen Einfluss auf das Körpergewicht. Eine genomweite Assoziationsstudie mit 249796 Personen konnte 32 Adipositas-Genorte identifizieren, die mit dem BMI assoziiert waren. Diese Varianten hatten zumeist sehr kleine Effektstärken und konnten insgesamt nur 5\% der BMI-Varianz erklären [Speliotes et al., 2010].

Sicher kann die wachsende Prävalenz von Übergewicht und Adipositas in der westlichen Welt nicht hinreichend durch Genetik erklärt werden. Vielmehr ist sie auf einen zunehmend körperlich inaktiven Lebensstil und die permanente Verfügbarkeit hochkalorischer Nahrungsmittel zurückzuführen. Das Verhalten des Einzelnen wird ganz entscheidend von den Verhältnissen, in denen wir leben, beeinflusst. Erst in den letzten 50 Jahren hat sich der ursprüngliche evolutionäre Vorteil der Fähigkeit, Fettreserven anzulegen, zu einem Nachteil gewandelt. Der vorherrschende andauernde Überfluss war in unserer evolutionären Programmierung nicht vorgesehen. Scheinbar ist der menschliche Körper im Sinne der Lebenssicherung eher auf Nahrungsmangel als auf Nahrungsüberschuss ausgerichtet. So gesehen könnte man die steigende Prävalenz der Adipositas auch als ganz normale Reaktion des Körpers auf eine für ihn unnormale Umwelt interpretieren und nicht als Pathologie eines Regulationssystems. Die biologische Gewichtskontrolle (set point) wird also durch Effekte der Umwelt deutlich überschattet [Berthoud und Morrison, 2008]. Unter den heutigen Bedingungen ist demzufolge die kognitive Kontrolle über die Nahrungsaufnahme weitaus wichtiger als die biologische Kontrolle (from instinct to intellect) [Müller MJ et al., 2010].

\section{Wie erfolgreich sind Gewichtsreduktionsprogramme?}

\section{Konservative Gewichtsreduktionsprogramme}

Trotz der Vielzahl an Diätbüchern und kommerziellen sowie ärztlich angeleiteten Gewichtsreduktionsprogrammen steigt die Zahl der Adipösen weltweit an [Haslam und James, 2005]. Gewichtsreduktion wäre einfach, da es nur eine Verhaltensänderung - heute oft Lebensstiländerung genannt - erfordern würde, um Kalorienreduktion und Bewegungssteigerung 
zu erreichen. Die Realität zeigt jedoch, dass die meisten Programme leider nur einen mäßigen Gewichtsverlust bewirken und zudem das reduzierte Gewicht von den meisten Betroffenen nicht gehalten werden kann [Shai et al., 2008; Gardner et al., 2007; Bischoff et al., 2012]. Shai et al. [2008] verglichen eine mediterrane Diät mit einer «low-fat»- und einer «lowcarbohydrate»-Diät bei 322 Personen mit einem durchschnittlichen BMI von $31 \mathrm{~kg} / \mathrm{m}^{2} ; 86 \%$ der Teilnehmenden waren Frauen, das durchschnittliche Alter betrug 52 Jahre. Die Ernährungsumstellung erfolgte über einen Zeitraum von 2 Jahren. Bei den 272 Teilnehmenden, die die Studie abgeschlossen hatten, lagen die durchschnittlichen Gewichtsverluste bei $3,3 \mathrm{~kg}$ (low-fat), 4,6 kg (mediterran) und 5,5 kg (low-carbohydrate); ein signifikanter Unterschied zwischen den Diätgruppen bestand nicht. Der maximale Gewichtsverlust wurde nach 6 Monaten erreicht, gefolgt von einer langsamen Gewichtswiederzunahme trotz weiterer Ernährungsumstellung. Ungeachtet der geringen Gewichtsreduktion fanden die Autoren einen positiven Effekt auf Fett- und Glukosestoffwechsel. In einer ähnlichen Studie verglichen Gardner et al. [2007] 4 kommerzielle Gewichtsreduktionsprogramme mit unterschiedlicher Zusammensetzung von Fett und Kohlenhydraten (Atkins ${ }^{\circledR}$, Zone $^{\circledR}$, Ornish ${ }^{\circledR}$ und LEARN ${ }^{\circledR}$ ) bei 311 Frauen mit einem BMI zwischen 27 und $40 \mathrm{~kg} / \mathrm{m}^{2}$. Nach 12 Monaten lag der durchschnittliche Gewichtsverlust bei 4,7 kg (Atkins), 1,6 kg (Zone), 2,6 kg (LEARN) und 2,2 kg (Ornish). Auch in dieser Studie wurde eine Besserung der metabolischen Risikofaktoren festgestellt. Da sich keine Diätstrategie als überlegen erwiesen hat, empfehlen die neuen S3-Leitlinien der Deutschen AdipositasGesellschaft (DAG) (www.deutsche-adipositas-gesellschaft.de) Ernährungsformen, die über einen ausreichenden Zeitraum zu einem Energiedefizit führen und keine Gesundheitsschäden hervorrufen. Um ein Energiedefizit zu erreichen, können verschiedene Ernährungsstrategien, je nach Vorliebe der Patienten, verwendet werden:

- Reduktion des Fettverzehrs

- Reduktion des Kohlenhydratverzehrs

- Reduktion des Fett- und Kohlenhydratverzehrs

Eine Energiereduktion von ca. $500 \mathrm{kcal}$ kann beispielsweise durch die Reduktion der Fettaufnahme auf ca. 60 g/Tag bei nicht begrenztem Verzehr von Kohlenhydraten erzielt werden. Damit ist eine Gewichtssenkung von durchschnittlich 3,2-4,3 $\mathrm{kg}$ in einem Zeitraum von 6 Monaten möglich. Extrem einseitige Ernährungsformen (z.B. totales Fasten oder Crash-Diäten) sind wegen hoher medizinischer Risiken und fehlendem Langzeiterfolg nicht ratsam. Explizit empfohlen wird der Verzicht auf Fast Food, Alkohol und zuckerhaltige Softdrinks.

Grundlage jedes Gewichtsmanagements sollte ein Basisprogramm sein, das die Komponenten Ernährungs-, Bewegungs- und Verhaltenstherapie umfasst. Neben einer realistischen Änderung der Ernährungsgewohnheiten wird daher angeregt, sich regelmäßig körperlich zu bewegen und sitzende Tätigkeiten zu begrenzen. Es sollten vor allem ausdauerorientierte Bewegungsformen empfohlen werden. Insgesamt soll- ten die Therapieziele realistisch sein und an individuelle Bedingungen angepasst werden. Dabei sollten individuelle Komorbiditäten, Risiken, Erwartungen und Ressourcen des Patienten stärker berücksichtigt werden und nicht nur die alleinige Gewichtsreduktion im Fokus stehen. Die meisten dieser Empfehlungen der Leitlinien haben einen hohen Empfehlungsgrad.

Eine Therapie mit Formulaprodukten sollte dagegen weitgehend Personen mit deutlicher Adipositas (BMI $\geq 35 \mathrm{~kg} / \mathrm{m}^{2}$ ) vorbehalten bleiben, bei denen eine größere Gefährdung besteht und daher eine größere Gewichtsabnahme wünschenswert ist. Allerdings kommt es gerade nach Beendigung eines solchen Therapieprogramms meist zu einem deutlichen Wiederanstieg des Körpergewichts - oft bis zum Ausgangsgewicht (Jo-Jo-Effekt) oder sogar darüber. In einer deutschen Studie mit 4850 Teilnehmenden eines 1-jährigen Programms, das eine Formuladiät über 12 Wochen anbietet (OPTIFAST52 ${ }^{\circledR}$ ) [Bischoff et al., 2012], wurde ein Gewichtsverlust bei Frauen von durchschnittlich 19,6 kg und bei Männern von durchschnittlich $26,0 \mathrm{~kg}$ festgestellt. Für eine vergleichsweise kleine Subgruppe von 301 Personen lagen auch Gewichtsdaten 2 Jahre nach Beendigung des Programms vor. Die Wiederzunahme war deutlich, das durchschnittliche Gewicht lag am Ende der 2-Jahres-Katamnese nur 5,9 kg unter dem Ausgangsgewicht; das entspricht einem Gewichtsverlust von 4,2\% des ursprünglichen Körpergewichts. Bei einem AusgangsBMI von $40,1 \mathrm{~kg} / \mathrm{m}^{2}$ in dieser Subgruppe ist das Langzeitergebnis als unbefriedigend einzustufen, und es ist unklar, wie groß der gesundheitliche Nutzen für die Teilnehmer ist.

Interessante Ergebnisse zu Langzeiterfolgen von Gewichtsreduktionsprogrammen bietet auch die Look AHEAD-Studie, die den Effekt einer intensiven Lebensstilintervention (ILI) im Vergleich zu einer Kontrollkondition auf die kardiovaskuläre Morbidität und Mortalität bei übergewichtigen und adipösen Patientinnen und Patienten mit Diabetes mellitus Typ 2 untersucht hat [Look AHEAD Research Group, 2014]. 5145 Patienten wurden randomisiert, $60 \%$ waren Frauen, das durchschnittliche Alter betrug 58 Jahre, der BMI lag bei $\geq 25$ $\mathrm{kg} / \mathrm{m}^{2}$. Trotz weiterführender Behandlung nahmen die Patienten nach dem ersten Therapiejahr langsam wieder an Gewicht zu. Nach 8-jähriger Behandlung mit ILI (1-2 individuelle Kontakte pro Monat, regelmäßige Gruppensitzungen, Möglichkeit der kostenfreien Nutzung von Formulaprodukten) lag das Gewicht um 4,7\% unterhalb des Ausgangsgewichts, in der Kontrollgruppe waren es $2,1 \%$. 50,3\% der Teilnehmenden in der ILI-Gruppe und 35,7\% in der Kontrollkondition hatten nach 8 Jahren 5\% oder mehr des Ausgangsgewichts verloren und 26,9 bzw. $17,2 \%$ mehr als $10 \%$. Diese Befunde werden von den Autoren sehr optimistisch als klinisch bedeutender Gewichtsverlust interpretiert. Tatsächlich kann bei einem 5-10\%igen Gewichtsverlust mit einer Verbesserung metabolischer Parameter gerechnet werden. Dennoch scheint der intensive und vor allem andauernde therapeutische Aufwand einen Gewichtsverlust von oft nur wenigen Kilogramm zu erzielen. Of- 
fensichtlich kann bei vielen Patienten mit den gängigen Gewichtsreduktionsprogrammen keine nachhaltige substanzielle Lebensstiländerung und Gewichtsstabilisierung erreicht werden. Ein erneuter Gewichtsanstieg scheint umso schneller zu erfolgen, je ausgeprägter die Gewichtsabnahme war, wobei der Erfolg bei Patienten mit stärkeren Gewichtsverlusten dennoch insgesamt höher zu sein scheint [Thomas et al., 2014]. Häufige Gewichtsschwankungen in der Vergangenheit stellen einen negativen Prädiktor für den Gewichtserhalt dar. Als positiver Prädiktor für den Gewichtserhalt wird immer wieder die regelmäßige Durchführung von Gewichtskontrollen bei gleichzeitig kontrolliertem bzw. restriktivem Essverhalten betont [Thomas et al., 2014]. Auch zahlreiche psychologische Faktoren und Verhaltensaspekte sind als Moderatoren und Mediatoren für eine erfolgreiche Gewichtsstabilisierung untersucht worden und scheinen eine nicht unerhebliche Rolle für die Fähigkeit zu spielen, ein reduziertes Gewicht erfolgreich zu halten bzw. zu stabilisieren [de Zwaan, 2013].

In der Zusammenschau kristallisieren sich 2 Probleme der derzeitigen Behandlung von Adipositas heraus. Erstens resultieren die meisten Therapieprogramme in eher geringen Gewichtsreduktionen und zweitens sind die Gewichtsreduktionen bei vielen Betroffenen nicht nachhaltig. Deswegen bedarf es einer realistischen Einschätzung der zu erwartenden Langzeiteffekte, wobei auch kleinere anhaltende Gewichtsabnahmen positiv beurteilt werden sollten.

\section{Adipositas-Chirurgie}

Im Gegensatz zu konservativen Programmen zeitigt Adipositas-Chirurgie beim überwiegenden Teil der Patienten eine längerfristige signifikante Gewichtsabnahme. Der Behandlungserfolg kann anhand des sogenannten excess weight loss beurteilt werden. Damit ist der prozentuale Anteil des Übergewichtsverlusts bezogen auf einen BMI von $25 \mathrm{~kg} / \mathrm{m}^{2}$ gemeint. Die substanzielle Gewichtsreduktion ist in der Regel mit einer langfristigen Verbesserung oder besseren Kontrollierbarkeit der Adipositas-assoziierten Komorbidität verbunden. In der weltweit längsten Katamnesestudie zur bariatrischen Chirurgie - der Swedish Obese Subject (SOS)-Studie wurde neben Effekten auf die Gewichtsabnahme auch eine 24\%ige Reduktion der Mortalität 15 Jahre nach der Operation gefunden [Sjöström et al., 2007].

Laut S3-Leitlinie zur Prävention und Therapie der Adipositas (www.deutsche-adipositas-gesellschaft.de) ist eine bariatrische Operation ab einem BMI von $40 \mathrm{~kg} / \mathrm{m}^{2}$ angezeigt oder aber ab einem BMI von mindestens $35 \mathrm{~kg} / \mathrm{m}^{2}$ bei gleichzeitigem Vorliegen Adipositas-assoziierter somatischer Erkrankungen. Ein weiteres Indikationskriterium stellt der Nachweis wiederholter erfolgloser konservativer Gewichtsreduktionsversuche dar [Runkel et al., 2011]. Präoperative Patienten in Deutschland weisen im Mittel einen BMI von $50 \mathrm{~kg} / \mathrm{m}^{2}$ auf.

Obwohl die Operationen heute laparoskopisch durchgeführt werden und die postoperative Mortalitätsrate mit weniger als $1 \%$ angegeben wird, handelt es sich doch um operative
Eingriffe, die nur teilweise rückgängig zu machen sind und deren Indikation daher gut geprüft werden muss. Zudem ist zu beachten, dass nicht nur prä- sondern gerade auch postoperativ von den Patienten eine hohe Adhärenz und Selbstfürsorge gefordert ist, um z.B. die notwendige lebenslange Substitution mit Vitaminen und Mineralstoffen (vor allem nach Magenbypass) zu gewährleisten. Studien haben ergeben, dass präbariatrische Patienten häufig an psychischen Störungen leiden mit aktuellen Prävalenzraten von bis zu 50\% und Lebenszeitprävalenzraten von bis zu 70\% [Mühlhans et al., 2009]. Daher wird häufig diskutiert, ob adipöse Patienten mit komorbiden psychischen Problemen von einer AdipositasChirurgie ausgeschlossen werden sollten. Allerdings verdeutlichen empirische Daten, dass dieses Vorgehen nicht gerechtfertigt scheint und dass psychische Störungen keine generellen Kontraindikationen für eine bariatrische Operation darstellen. Neben der bereits erwähnten Verbesserung somatischer Begleiterkrankungen zeigt sich nach AdipositasChirurgie nämlich in der Regel auch eine deutliche Verbesserung des psychischen Befindens. Meistens kommt es nach der Operation und im Zuge der Gewichtsabnahme zur Stabilisierung sozialer Beziehungen, Reduktion des Krankenstands und Verbesserung der Erwerbsfähigkeit. Auch depressive Störungen, Angsterkrankungen und Essstörungen verringern sich überwiegend [de Zwaan et al., 2010; 2011a]. Ob diese Erfolge auch langfristig gehalten werden können, werden die gerade laufenden Langzeitstudien zeigen (Longitudinal Assessment of Bariatrc Surgery, LABS; Essen-Bochumer Obesity Treatment Study, EBOTS).

\section{Spezielle Programme zur Gewichtsstabilisierung}

Nachhaltige Gewichtsreduktionen bedürfen entweder innovativer Strategien zur Gewichtsstabilisierung nach erfolgter Gewichtsabnahme oder langfristig angelegter Nachsorgekonzepte. Letztere sollten Techniken zur Verbesserung von Selbstkontrolle und Rückfallprophylaxe vermitteln und könnten niederfrequent angeboten werden. Allerdings scheint die Motivation zur regelmäßigen Teilnahme an solchen Angeboten eher begrenzt zu sein. Dies könnte daran liegen, dass diese auf Gewichtsstabilisierung ausgerichteten Nachsorgeprogramme als weniger belohnend erlebt werden als reine Gewichtsreduktionsprogramme. Die Einstellung der Betroffenen demgegenüber kann sicherlich nur geändert werden, wenn die im Gesundheitssystem Tätigen der Gewichtsstabilisierung einen höheren Stellenwert beimessen.

In der Literatur finden sich zunehmend evaluierte Programme zur Gewichtsstabilisierung, die an teilweise großen Patientengruppen eingesetzt wurden [Svetkey et al., 2008; Wing et al., 2006]. In einer großen multizentrischen Studie verglichen Svetkey et al. [2008] monatlichen persönlichen Kontakt, Zugang zu einer interaktiven Webseite und eine minimale Intervention mit schriftlichen Empfehlungen. Es wurden 1032 Personen eingeschlossen, die in einem vorgeschalteten 6-monatigen Gewichtsreduktionsprogramm zumindest 
$4 \mathrm{~kg}$ abgenommen hatten. In allen 3 Gruppen nahmen die Patienten wieder an Gewicht zu. Nach 30 Monaten lag die Gewichtszunahme in der Gruppe mit persönlichem Kontakt bei $4,0 \mathrm{~kg}$, in der Gruppe mit Zugang zur Webseite bei 5,2 kg und in der Gruppe mit Minimalintervention bei $5,5 \mathrm{~kg}$. Insgesamt blieben $71 \%$ der Teilnehmer unter dem Eingangsgewicht und $37 \%$ der Teilnehmer 5\% oder mehr unterhalb des Gewichts zum Zeitpunkt der Randomisierung. In einer ähnlichen Studie verglichen Wing et al. [2006] persönlichen Kontakt, eine internetbasierte Intervention und eine Minimalintervention mit monatlichen Newslettern. Es wurden 314 Personen eingeschlossen, die in den letzten beiden Jahren im Durchschnitt $19,3 \mathrm{~kg}$ abgenommen hatten. Auch in dieser Studie zeigte sich, dass eine Gewichtswiederzunahme nicht verhindert werden konnte. Die Gewichtszunahmen in den 3 Gruppen betrugen nach 18 Monaten 2,5 kg (persönlicher Kontakt), 4,7 kg (Internet) und 4,9 kg (Minimalintervention). Tägliches Wiegen war mit einer geringeren Gewichtszunahme assoziiert. In beiden Programmen waren die Patienten, die persönlichen Kontakt zu einem Therapeuten hatten, erfolgreicher, unabhängig davon, ob es sich um einen persönlichen Kontakt oder Kontakt über das Internet handelte. Wichtig scheint vor allem der Kontakt an sich zu sein und weniger das Medium. Die optimale Kontaktfrequenz ist allerdings noch nicht eindeutig ermittelt worden (z.B. monatlich oder wöchentlich). Middleton et al. [2012] berichteten in einer Metaanalyse von insgesamt 11 Gewichtserhaltungsstudien über einen zusätzlichen $\mathrm{Ge}$ wichtsverlust von 3,2 kg über 17,6 Monate nach Einführung eines Programms zur Gewichtsstabilisierung und eine eher mäßige Effektstärke von 0,385 (kombiniert über alle 11 Studien). $\mathrm{Zu}$ beachten ist, dass Gewichtsstabilisierungskonzepte im Gegensatz zu Gewichtsreduktionsprogrammen nicht auf Gewichtsabnahme fokussieren [de Zwaan, 2013]. Stattdessen werden Strategien zur Verbesserung von Selbstakzeptanz, positivem Körperbild und Umgang mit gewichtsbezogener Diskriminierung vermittelt. Dabei spielen der persönliche Kontakt zum Behandlerteam, regelmäßige Selbstbeobachtung, das Erarbeiten von Problemlösestrategien und die Rückfallprophylaxe eine wichtige Rolle. Ebenso ist soziale Unterstützung bedeutsam, um die gewünschten Verhaltensänderungen hinsichtlich Ernährung und Bewegung zu erreichen.

Die regelmäßige Teilnahme stellt einen wichtigen Prädiktor für den Erfolg dar; dies schließt auch die regelmäßige Nutzung von Internetangeboten mit ein [Neve et al., 2010].

\section{Psychische Komorbidität}

\section{Depression}

Entgegen früherer Befunde lassen aktuelle bevölkerungsbasierte Studien ein häufigeres Auftreten von Depressionen und Angsterkrankungen bei adipösen Menschen im Vergleich $\mathrm{zu}$ normalgewichtigen Personen vermuten. Die höhere psychische Komorbidität scheint vorrangig an die Höhe des BMI
(Adipositas Grad I und II) und das weibliche Geschlecht gekoppelt zu sein [Mühlhans und de Zwaan, 2008].

Eine Metaanalyse von prospektiven, longitudinalen Studien ergab folgenden reziproken Zusammenhang: Bei depressiven Personen besteht ein erhöhtes Risiko für Adipositas (Odds Ratio 1,58) und vice versa haben adipöse Menschen ein erhöhtes Risiko für Depressionen (Odds Ratio 1,55) [Luppino et al., 2010]. Möglicherweise gibt es für diese Zusammenhänge biologische Erklärungen. Beispielsweise könnte die Entwicklung einer depressiven Erkrankung durch Adipositas-typische Aspekte wie inflammatorische Prozesse, Insulinresistenz oder eine HPA-Achsen-Dysfunktion gefördert werden. Ebenso könnten Psychopharmaka (z.B. bestimmte Antidepressiva) zur Gewichtserhöhung beitragen. Potenziell können auch Diskriminierungserfahrungen oder die mangelnde Selbstfürsorge die reziproke Beziehung zwischen Depression und Adipositas erklären. Ein weiterer Zusammenhang ergibt sich aus der Tatsache, dass Nahrungsaufnahme nicht nur der Sättigung, sondern oft auch der Affektregulation dient.

\section{Essstörungen}

Adipositas stellt per se keine Essstörung dar. Essstörungen, hier vor allem die Binge-Eating-Störung (BES), findet man bei adipösen Menschen jedoch doppelt so häufig im Vergleich zu Normalgewichtigen. Mittlerweile wurde die BES dank umfassender, langjähriger Forschungsarbeiten als eigenständige Diagnose im DSM-5 aufgenommen [APA, 2013]. Übergewichtige Frauen mit BES unterscheiden sich in mehreren Aspekten von übergewichtigen Frauen ohne BES. Sie berichten meistens ein höheres Gewicht und auch einen früheren Beginn von Übergewichtigkeit sowie mehr Gewichtsschwankungen. Hinzu kommen eine ausgeprägte Überbewertung von Gewicht und Körper, verbunden mit Unzufriedenheit, Selbstwertkonflikten und einem höheren Ausmaß psychischer Probleme, worin sie Patientinnen mit Bulimia nervosa ähneln. Psychotherapie hat sich mittlerweile als Therapie der ersten Wahl bei der Behandlung einer BES erwiesen, wobei die meiste Evidenz für kognitiv-verhaltenstherapeutische (KVT) Programme vorliegt. Alternativen stellen die interpersonelle Therapie (IPT), dialektische Verhaltenstherapie (DVT) oder auch die psychodynamische Therapie dar. Essanfälle remittieren in der Regel wesentlich besser als bei Patientinnen mit Bulimie. Neben Psychotherapie hat auch die psychopharmakologische Behandlung einen festen Platz und gilt als Therapie zweiter Wahl. Obwohl noch kein Medikament für die Behandlung einer BES zugelassen wurde, haben sich Selektive Serotonin-Wiederaufnahmehemmer (SSRIs) und Serotonin-NoradrenalinWiederaufnahmehemmer (SNRIs) als wirksam erwiesen (OffLabel-Behandlung). Einschränkend muss erwähnt werden, dass Rückfälle (vor allem Essanfälle) nach Absetzen des Medikaments oder bei Erhaltungstherapie häufig sind, was auf eher geringe bzw. ungewisse Langzeiterfolge hindeutet.

Gewichtsreduktionsprogramme, die mit starker kalorischer Einschränkung arbeiten, zeitigen nicht nur eine Gewichtsab- 
nahme, sondern auch eine Verringerung der Essattacken, was den ursprünglichen Annahmen entgegensteht [de Zwaan et al., 2005]. Möglicherweise ist dieser Effekt darauf zurückzuführen, dass viele dieser Programme Strategien vermitteln, die sich mit KVT-Ansätzen überlappen. Dazu zählen z.B. eine regelmäßige Mahlzeitenstruktur und die Anwendung von Stimulus-Kontrolltechniken. Unklar ist noch, ob Adipositas und BES gleichzeitig oder besser nacheinander behandelt werden sollten. Die bisherige Erfahrung hat gezeigt, dass die primäre psychotherapeutische Behandlung einer BES sowohl die Essstörungssymptomatik als auch die psychische Befindlichkeit verbessert und damit auch einem weiteren Gewichtsanstieg entgegenwirkt. Gleichwohl resultiert sie nicht in einer substanziellen Gewichtsreduktion, worüber die Patienten informiert werden sollten, um Enttäuschungen vorzubeugen. Auch die Therapieabbruchsraten unterscheiden sich nicht zwischen adipösen Patienten mit und ohne BES. Allerdings haben Patientinnen, die nach einer Gewichtsreduktion weiterhin oder wieder die Kriterien für eine BES erfüllen, einen schlechteren Langzeitverlauf des Gewichts.

Neben der BES sind noch weitere maladaptive Facetten pathologischen Essverhaltens bekannt. Dazu gehört das sogenannte Grasen (grazing), bei dem Betroffene über einen längeren Zeitraum ständig kleinere Nahrungsmengen zu sich nehmen. Dabei handelt es sich vornehmlich um Süßigkeiten oder Snacks. Obwohl «grazing» in der klinischen Routine bei übergewichtigen und adipösen Personen häufig gesehen wird, sind bislang noch keine diagnostischen Kriterien definiert, die diese Variante gestörten Essverhaltens hinreichend operationalisieren. Hingegen ist das das «Night Eating Syndrome» (NES), eine andere Form essgestörten Verhaltens, mittlerweile im DSM-5 gelistet [APA, 2013].

\section{Aufmerksamkeitsdefizit/Hyperaktivitätsstörung (ADHS)}

Adipositas geht mit einer erhöhten Impulsivität und exekutiven Dysfunktionen einher [Müller A et al., 2010]. Sowohl bei Kindern und Jugendlichen als auch bei Erwachsenen besteht eine Assoziation zwischen Adipositas und ADHS. In einer repräsentativen deutschen Bevölkerungsstichprobe lag die Prävalenz von ADHS im Erwachsenenalter bei Personen mit Adipositas mit 9,7\% signifikant höher als bei Übergewichtigen $(4,5 \%)$ und Normalgewichtigen (4,1\%) [de Zwaan et al., 2011b]. Bei Teilnehmern an Gewichtsreduktionsprogrammen wird eine ADHS-Häufigkeit von 25-40\% angegeben [Fleming et al., 2005; Cortese et al., 2008; Pagoto et al., 2009]. Es gibt erste Hinweise, dass Patienten mit ADHS weniger an Gewicht abnehmen [Levy et al., 2009]. Die Beeinträchtigung der Aufmerksamkeit und die starke Impulsivität und Unruhe scheinen sich negativ auf die Fähigkeit auszuwirken, über längere Zeit Kontrolle über das Essverhalten ausüben zu können. Kinder und Jugendliche unter angemessener medikamentöser Therapie mit z.B. Methylphenidat können ihr Essverhalten besser kontrollieren [Erhart et al., 2012]. Auch bei Erwachsenen mit ADHS kann Methylphenidat den Gewichts- erhalt verbessern. Die routinemäßige Medikation setzt jedoch in jedem Fall eine professionelle Diagnostik der ADHS voraus. Daneben ist ein zuverlässiges Monitoring der potenziellen Nebenwirkungen gerade bei adipösen Menschen unbedingt notwendig.

Ausgeprägte psychische Probleme wie ADHS, Essanfälle (binge eating) oder Depression sollten speziell behandelt werden, da sie den Gewichtserhalt unabhängig von biologischen und Umweltfaktoren beeinträchtigen können [de Zwaan, 2013].

\section{Stigmatisierung}

Es wird davon ausgegangen, dass Adipositas in unserer Gesellschaft mehr stigmatisiert wird als andere körperliche und seelische Erkrankungen. Stigmatisierung und Diskriminierung von adipösen Menschen findet in zahlreichen Kontexten statt: in den Medien, in der Allgemeinbevölkerung, durch Personalentscheider und selbst Personen im Gesundheitswesen. Auch ehemals adipöse Menschen erleben weiterhin Stigmatisierung. In $2 \mathrm{sehr}$ ähnlichen Untersuchungen in der deutschen Allgemeinbevölkerung (2005 und 2012) wurde die Subskala «Weight Control Blame» des Antifat-AttitudeTests jeweils 1000 Personen vorgegeben [Hilbert et al., 2008; Ellrott, 2012]. In beiden Untersuchungen gaben $23 \%$ eine stigmatisierende Einstellung Adipösen gegenüber an. Dabei scheinen Überzeugungen, dass Adipöse aufgrund von Faulheit, Willensschwäche oder Disziplinlosigkeit allein verantwortlich für ihr hohes Gewicht sind, weit verbreitet zu sein [Sikorski et al., 2011]. Die Stigmatisierung stieg mit zunehmendem Alter und geringerer Schulbildung. Frauen gaben mehr und Teilnehmer aus den neuen Bundesländern weniger stigmatisierende Einstellungen an. In einer Untersuchung mit 447 Psychologiestudenten, die Fallvignetten beurteilen sollten, konnten Ebneter und Latner [2013] zeigen, dass Personen mit Adipositas und Personen mit BES ein signifikant höheres Ausmaß an mangelnder Selbstkontrolle zugesprochen wird als Personen mit Anorexia nervosa, Bulimia nervosa und auch als Personen mit Depression.

Stigmatisierungserfahrungen können zu Selbstinvalidierung führen. Im Sinne eines Circulus vitiosus kann es zu einer Zunahme maladaptiven Essverhaltens kommen. Wenn Stigmatisierung im Gesundheitswesen auftritt, können Prävention und Behandlung erschwert werden. Zur Wirksamkeit von Anti-Stigma-Programmen kann derzeit keine klare Aussage gemacht werden.

\section{Fazit}

Übergewicht und Adipositas zeigen eine immer noch steigende Prävalenz, vor allem in der westlichen Welt. Adipositas geht nicht nur mit einer hohen organischen, sondern auch psychischen Komorbidität (z.B. Depression, Essstörungen) 
sowie ausgeprägter Stigmatisierung einher. Gewichtsreduktionsansätze sind kurzfristig effektiv, ein Gewichtserhalt ist jedoch nur schwer möglich. Eine Aufrechterhaltung von nur 5\% Verlust des Ausgangsgewichts wird von vielen Autoren als Erfolg gesehen, bei längerfristigen und intensiven Programmen scheinen etwa 50\% der Teilnehmer dieses Ziel erreichen zu können. Spezielle Ansätze zur Gewichtsstabilisierung zeigen keine besseren Erfolge. Selbst geringe Gewichts- abnahmen scheinen die metabolischen Parameter jedoch positiv beeinflussen zu können. Bei Adipositas Grad III muss eine chirurgische Maßnahme in Betracht gezogen werden.

\section{Disclosure Statement}

Bei den Autorinnen besteht kein Interessenskonflikt.

\section{Literatur}

American Psychiatric Association (APA): Diagnostic and Statistical Manual of Mental Disorders, 5th Edition. Arlington, APA, 2013.

Berthoud HR, Morrison C: The brain, appetite, and obesity. Annu Rev Psychol 2008;59:55-92.

Bischoff SC, Damms-Machado A, Betz C, et al.: Multicenter evaluation of an interdisciplinary 52-week weight loss program for obesity with regard to body weight, comorbidities and quality of life - a prospective study. Int J Obes (Lond) 2012;36:614-624.

Cortese S, Angriman M, Maffeis C, et al.: Attentiondeficit/hyperactivity disorder (ADHD) and obesity: a systematic review of the literature. Crit Rev Food Sci Nutr 2008;48:524-537.

de Zwaan M: Könnten operative Eingriffe die Psychopathologie langfristig besser beeinflussen als evidenzbasierte Psychotherapie? Pro und Contra bariatrischer Chirurgie in der Adipositasbehandlung. Verhaltenstherapie 2012;22:199-203.

de Zwaan M: Gewichtsstabilisierung nach konservativer und bariatrischer Therapie; in Wirth A, Hauner H (eds): Adipositas - Ätiologie, Folgekrankheiten, Diagnostik, Therapie, ed 4. Berlin, Springer, 2013, pp 319-325.

de Zwaan M, Enderle J, Wagner S, et al. :Anxiety and depression in bariatric surgery patients: a prospective, follow-up study using structured clinical interviews. J Affect Disord 2011a;133:61-68

de Zwaan M, Gruß B, Mueller A, et al.: Association between obesity and adult attention-deficit/hyperactivity disorder (ADHD) in a German community-based sample. Obes Facts 2011b;4:204-211.

de Zwaan M, Hilbert A, Swan-Kremeier L, et al. Comprehensive interview assessment of eating behavior 18-35 months after gastric bypass surgery for morbid obesity. Surg Obes Relat Dis 2010;6:79-85

de Zwaan M, Mitchell JE, Mussell MP, et al.: Shortterm cognitive behavioral treatment does not improve long-term outcome of a comprehensive verylow-calorie diet program in obese women with binge eating disorder. Behav Ther 2005;36:89-99.

Ebneter DS, Latner JD: Stigmatizing attitudes differ across mental health disorders: a comparison of stigma across eating disorders, obesity, and major depressive disorder. J Nerv Ment Dis 2013;201:281285.

Ellrott T: Stigmatisierung von Adipositas in Deutschland, Ergebnisse einer repräsentativen Befragung im Sommer 2012. Vortrag im Rahmen der 28. Jahrestagung der Deutschen Adipositas-Gesellschaft (DAG) e.V. in Stuttgart am 6. Oktober 2012.

-Erhart M, Herpertz-Dahlmann B, Wille N, et al.: Examining the relationship between Attention-Deficit/Hyperactivity Disorder and overweight in children and adolescents. Eur Child Adolesc Psychiatry 2012;21:39-49.
Fleming JP, Levy LD, Levitan RD: Symptoms of attention deficit hyperactivity disorder in severely obese women. Eating Weight Disord 2005;10:10-13.

Frieling H, Hinney A, Bleich S: Genetische Aspekte der Adipositas; in Herpertz S, de Zwaan M, Zipfel $S$ (eds): Handbuch der Essstörungen und Adipositas. Heidelberg, Springer, 2008, pp 265-270.

Gardner CD, Kiazand A, Alhassan S, et al.: Comparison of the Atkins, Zone, Ornish, and LEARN diets for change in weight and related risk factors among overweight premenopausal women: the A TO Z Weight Loss Study: a randomized trial. JAMA 2007;297:969-977.

Haslam DW, James WP: Obesity. Lancet 2005;366: 1197-1209.

Hilbert A, Rief W, Braehler E: Stigmatizing attitudes toward obesity in a representative populationbased sample. Obesity 2008;16:1529-1534.

Knerr I, Topf HG, Hablawetz B, et al.: Frühe Einflussfaktoren auf das Körpergewicht und die Prävalenz von Übergewicht zum Zeitpunkt der Schuleingangsuntersuchung bei 4610 Kindern im Raum Erlangen/ Nordbayern. Gesundheitswesen 2005;67:183-188.

Kurth BM: Erste Ergebnisse der «Studie zur Gesundheit Erwachsener in Deutschland» (DEGS). Bundesgesundheitsbl 2012;55:980-990.

Levy LD, Fleming JP, Klar D: Treatment of refractory obesity in severely obese adults following management of newly diagnosed attention deficit hyperactivity disorder. Int J Obesity 2009;33:326-334.

Look AHEAD Research Group: Eight-year weight losses with an intensive lifestyle intervention: The look AHEAD study. Obesity (Silver Spring) 2014; 22:5-13.

Luppino FS, de Wit LM, Bouvy PF, et al.: Overweight, obesity, and depression: a systematic review and meta-analysis of longitudinal studies. Arch Gen Psychiatry 2010;67:220-229.

Middleton KMR, Patidar SM, Perri MG: The impact of extended care on long-term maintenance of weight loss: a systematic review and meta-analysis. Obes Rev 2012;13:509-517.

Mühlhans B, de Zwaan M: Psychische Komorbidität bei Adipositas. Adipositas 2008;2:148-154.

Mühlhans B, Horbach T, de Zwaan M: Psychiatric disorders in bariatric surgery candidates: a review of the literature and results of a German prebariatric surgery sample. Gen Hosp Psychiatry 2009;31:414-421.

Müller MJ, Bosy-Westphal A, Heymsfield SB: Is there evidence for a set point that regulates human body weight? F1000 Med Rep 2010;2:59.
Müller A, Pauli E, de Zwaan M: Ist es doch Willenskraft? Exekutivfunktionen bei Adipositas. Adipositas 2010;3:31-37.

Neve M, Morgan PJ, Jones PR, Collins CE: Effectiveness of web-based interventions in achieving weight loss and weight loss maintenance in overweight and obese adults: a systematic review with meta-analysis. Obes Rev 2010;11:306-321.

Pagoto SL, Curtin C, Lemon SC, et al.: Association between adult attention deficit/hyperactivity disorder and obesity in the US population. Obesity 2009;17:539-544.

Rosario AS, Kurth BM, Stolzenberg H, Ellert U, Neuhauser $\mathrm{H}$ : Body mass index percentiles for children and adolescents in Germany based on a nationally representative sample (KiGGS 2003-2006). Eur J Clin Nutr 2010;64:341-349.

Runkel N, Colombo-Benkmann M, Hüttl TP, et al.: Bariatric surgery. Dtsch Arztebl Int 2011;108:341346.

Shai I, Schwarzfuchs D, Henkin Y, et al.: Dietary Intervention Randomized Controlled Trial (DIRECT) Group: Weight loss with a low-carbohydrate, Mediterranean, or low-fat diet. N Engl J Med 2008;359:229-241.

Sikorski C, Luppa M, Kaiser M, et al.: The stigma of obesity in the general public and its implications for public health - a systematic review. BMC Public Health 2011;11:661.

Sjöström L, Narbro K, Sjöström CD, et al.: Swedish Obese Subjects Study. Effects of bariatric surgery on mortality in Swedish obese subjects. N Engl J Med 2007:357:741-752.

-Speliotes EK, Willer CJ, Berndt SI, et al.: Association analyses of 249,796 individuals reveal 18 new loci associated with body mass index. Nat Genet 2010; 42:937-948.

Svetkey LP, Stevens VJ, Brantley PJ, et al.: Comparison of strategies for sustaining weight loss: the weight loss maintenance randomized controlled trial. JAMA 2008;299:1139-1148.

Thomas JA, Bond DS, Phelan S, Hill JO, Wing RR: Weight-loss maintenance for 10 years in the National Weight Control Registry. Am J Prev Med 2014;46:17-23.

Wing RR, Tate DF, Gorin AA, et al.: A self-regulation program for maintenance of weight loss. N Engl J Med 2006;355:1563-1571.

Wirth A: Ätiologie und Diagnostik der Adipositas; in Herpertz S, de Zwaan M, Zipfel S (eds): Handbuch der Essstörungen und Adipositas. Heidelberg, Springer, 2008, pp 246-254. 